\title{
To Risk and To Represent: Local and Global Disability in Context
}

I am excited to be the International Editor for the new journal 'Considering Disability: the Contemporary Studies Journal.' For me personally, it is a daunting new project and I am very aware of what is at stake in claiming to be an 'International Editor' of a journal that is interested and invested in Disability Studies. I come from a Middle Eastern Bedouin background, and I have Multiple Sclerosis. But I am not sure whether my identity as a woman comes first, or as a 'disabled' woman, or whether my racial background is the first determining factor in these so-called identity politics. How do I wish to be perceived? And, what is my personal responsibility towards myself and the community? How do I make sure that my identity (and that of other disabled individuals) is not reduced and even worse, obliterated? I am an academic scholar who is also struggling with a disability, yet my identity should not simply be a Disabled Scholar. I am also a woman, one who is also a hybrid, and is considered queer in every sense of the word. My identity is simply one that does not fall under the safe zone of normalcy. I am not a normate, yet I am still asking: how would I consciously choose to be perceived? What part of my identity should come first?

My aim as an International editor is to help shed light on silenced voices of individuals with disabilities. Generally speaking, non-Western experiences of disability and its perceptions and stigma are underrepresented, and often neglected, in the field of Disability Studies. There are many reasons for this absence of non-Western experiences of disability in academic discourse. Of course, culture plays a big role, and social stigma continues to haunt individuals with disabilities. It is not altogether surprising that women with disabilities who are considered 'racial others' are faced with double, if not triple, marginalization and are excluded from any axis of 'normalcy.' I am always concerned with the politics of a disabled female identity, an identity that cannot be separated from race, gender, and class.

'Considering Disability' does not aim to represent all experiences of disability, or a universal definition of disability, but rather attempts to consider the various ways that disability is experienced and perceived. We cannot offer one view and throughout our selection of material to be published, we will try to include different, diverse, and discrepant experiences that complement and contradict each other. As the International Editor, I am interested in the ways by which 'local' and 'global' experiences of disability shape people's lives. What are the social, cultural, and personal differences and commonalities between someone living with a disability or physical impairment in the UK, Africa, or the Middle East?

In essence, there is almost always a process of labeling and dehumanizing that takes place when one chooses to claim any identity, let alone a disabled 
identity. When you come out with a disability, you are claiming it - and that should be fine and liberating, right? Is claiming disability also oppressive? The question that presents itself is, does such a claim mean you have chosen to glorify one side of your identity? And if so, is disability to be glorified?

I have raised many questions and I cannot make any concluding statements. I urge for a dialogue that cuts across national frontiers, a dialogue that seeks to interrogate and to facilitate a deeper understanding of the operation of disability in different cultural and social contexts. Individual circumstances and cultural differences shape local worlds, but the dialogue between local and global is meant to enhance our understanding of the transference of disability identity models and whether this is indeed the case both in literary and cultural representations and perceptions of disability.

That said, in the broadest sense, the personal is the political. As International Editor, I start this journey of our journal's first issue on the premise that there is still so much to consider when thinking about disability; a global dialogue is long overdue, and the politics of representation are to be endlessly (yet fruitfully) probed.

\section{Dr. Shahd Alshammari}

Considering Disability International Editor 